\title{
Situated learning with Bebras tasklets
}

\author{
Carlo Bellettini ${ }^{10000-0001-8526-4790]}$, Violetta Lonati ${ }^{1}[0000-0002-4722-244 X]$, \\ Mattia Monga $^{1[0000-0003-4852-0067]}$, Anna Morpurgo ${ }^{10000-0003-0081-914 X]}$,' \\ and Martina Palazzolo ${ }^{2}$ \\ 1 Università degli Studi di Milano, Italy, http://aladdin.di.unimi.it \\ ${ }^{2}$ Istituto Comprensivo 'Ilaria Alpi', Milan, Italy
}

\begin{abstract}
A Bebras short task, a tasklet, is designed to provide a source for exploring a computational thinking concept: at the end of the contest it could be used as a starting point to delve deeper into a computing topic. In this paper we report an experience which aims at taking full advantage of the potential of Bebras tasklets. A math teacher asked her pupils to act as Bebras "trainers" for younger mates. The pupils, in pairs, were assigned to design and prepare a tangible game inspired by a Bebras tasklet, devised for the younger pupils to practice. They also had to explain the game to the younger pupils, make them play and support them in solving it. In carrying out this assignment the pupils acting as trainers had to deeply explore the Bebras tasklet and face its computational thinking challenge, and also practiced soft skills as collaborating with peers towards a common goal, adapting language and communicative style to engage with younger mates, devising and designing a tangible object, and planning its creation. The experience proved that using Bebras tasklets as the social and cultural context for situated learning of computational thinking competencies is indeed quite productive.
\end{abstract}

Keywords: computational thinking $\cdot$ situated learning $\cdot$ Bebras

\section{Introduction}

Bebras, the "International Challenge on Informatics and Computational Thinking, $3713[9$, is a popular initiative aimed at introducing to the fundamental concepts of informatics pupils from 1st grade to the end of secondary school, independently of their exposure to formal computer science studies. The challenge is organized on an annual basis in several (54 in 2018) countries since 2004, with almost three million participants in the last edition. The setting of the contest is slightly different in each country, but in general participants have to solve a set of about 10-15 tasks that are designed to be fun and attractive, adequate for the contestants' age, and solvable in an average time of three minutes, hence called tasklets. Moreover, since the contest is especially aimed at a non-vocational audience, tasklets should be independent of specific curricular activities and avoid

3 http://bebras.org/'Bebras' is the Lithuanian word for 'beaver': some countries translate it, and others use it as a brand name. 
the use of jargon. In fact, Bebras tasklets focus on that part of informatics that should become familiar to everyone, not just computing professionals. A number of tasklets are prepared every year by an international team of experts in computer science and computing education; then each national organization selects, adapts and translates a set of tasklets to be used in the local competition. After the contest, the translated tasklets used in the challenge are publicly released by most countries and can be used by teachers as teaching resources in their school practice 82 . In Italy, the challenge is proposed to teams of (up to) 4 pupils, divided into 5 categories according to contestant age (from 8 to 18 years), and administered by an online platform [34] that displays tasklets and collects the submitted answers. Once the contest is over, the same platform also allows pupils to access their answers and check the solutions. In particular, for each tasklet they can see the correct answer (or one of them, if applicable), compare their answer with the correct one, access the explanation on how the answer could be obtained, and read a short text ("It's informatics") about the informatics content of the tasklet. The system is available also beyond the contest, so that the challenge can be simulated and answers and explanation be accessed at any time. Usually, after the challenge pupils are eager to know whether the solutions they had submitted are right or wrong. However, when they have learnt they got an answer (partially) wrong, many are not too motivated to investigate further. During the last edition, we observed some pupils when the teacher disclosed the results, and they did not spend much time in examining the explanation or ascertaining they really understood the tasklet and the correct answer, even when the teacher urged them to. In order to encourage pupils' engagement, a math class teacher (author Martina Palazzolo) asked her 6th grade pupils to act as Bebras trainers for a primary school class (3rd grade); pupils were requested to design and prepare tangible games, inspired by Bebras tasklets, to be offered to younger pupils to practice in view of next year's Bebras Challenge. The trainers then had to explain the game to the younger pupils, make them play and support them in solving the tasklet. The last of these training sessions was observed by a computing education expert, member of the Italian Bebras Committee (author Violetta Lonati), whose observations were the basis for the assessment of the project. In this paper we report this experience and the related findings. In Section 2 we describe the activities that have been carried out and the project's learning goals and methodology. In Section 3 we present the tasklets chosen by the pupils and the derived games they built, and we report about the interaction of the pupils with their mates and their teacher during the preparation of games and the training sessions. In Section 4 we give some elements to evaluate the overall experience and we draw some conclusions.

\section{Description of the project}

In this section we present the project, and in particular the context where it was proposed, the planned activities, the learning goals, and the methodology used to manage the activities and assess the results. 
Context and activities The experience involved a 6 th grade class of a publiclyfunded school, composed by 22 pupils (age 10-11 years). This particular class of the school is involved in a three-year long experimentation on innovative teaching methods which involves all the class teachers. The class timetable schedules a two-hour weekly session of mildly structured activities devoted to the development of cross competencies, conducted by the math and science teacher (author Martina Palazzolo, from now on, the Teacher). This was the context where the project was conducted throughout the second term (January-May) of year 2019. All the pupils were familiar with Bebras tasklets since they had already played a simulation of the challenge in the first term. They had also spent a couple of hours working in groups to check their answers and study the explanations provided in the Italian Bebras Platform. At the beginning of the project pupils were informed by the Teacher that they were going to act as Bebras trainers for 3rd graders. From now on we will call these groups of pupils trainers and trainees, respectively. The project was thus organized in two phases: (1) Preparation of the games. Working in pairs, trainers were asked to choose a Bebras tasklet, design a tangible game, and build it (with cardboard, etc.) so that it would be possible for the younger pupils to try it without accessing the original tasklet through the platform. This phase lasted around three months. The game had to be built in such a way that it would be clearly accessible and understandable by a peer. In particular, the requirements were that the title, the text, and question should be clearly readable, the game should be self-explanatory and have all the elements necessary to solve it, and it should be equipped with an envelope containing the correct answer. It was not requested though that the game were accessible by a 3rd grader without some help or further explanation by the trainers. (2) Training sessions. Towards the end of the school year the trainers actually trained a $3 \mathrm{rd}$ grade class of 22 pupils by using their games. The trainees were invited to two training sessions, one held in April and one in May 2019, and played all the games. Between the two sessions, the Teacher conducted a discussion among the trainers: they were requested to report to their mates how the training sessions went and whether they thought their games needed some adjustments.

Soon after the project had started, a computing education expert and member of the national Bebras Committee (author Violetta Lonati, from now on the Expert) was asked by the Teacher to contribute to the project as a mentor and supervisor. The Expert's role was to support the Teacher in relation to the computational thinking aspects involved by the tasklets chosen by the pupils, and hence to help her understand how the project improved the learning of computational thinking skills. Teacher and Expert had already collaborated in other computing education projects in the past [5], after they had met several years ago in a refreshment course attended by the Teacher.

\subsection{Learning goals}

The project aimed at several learning goals for the trainers. On the one hand, the trainers were expected to improve in the computational thinking skills (CT) 
implied by Bebras tasklets [82]. In particular the focus was on the following CT skills.

Represent - representing information through abstraction such as models, diagrams, symbolic encodings and understanding such representations;

Algo_think - automating tasks through algorithmic thinking (i.e., series of ordered steps);

Implement - implementing algorithmic solutions complying with some predefined syntax (i.e., coding);

Organize - logically organizing data;

Reason - analytically reasoning about data, objects, situations to check properties and draw logical conclusions.

In stating such goals, we adopt the framework discussed in [2, mostly based on the the operational definition of CT [1] developed by ISTE (International Society for Technology in Education) and CSTA (Computer Science Teachers Association). Only some of the skills described in the paper are mentioned here, namely those that are relevant with respect to the set of tasklets chosen by the pupils (see Section 3). In fact, since each pair of trainers chose a different tasklet, they actually practiced different skills among the above ones. We should also mention here that different (and only partially overlapping) definitions of CT exist; a good recent survey can be found in [6], which discusses also frequent misconceptions of CT by primary teachers. On the other hand, the project also aimed at promoting soft skills like: learning to learn; collaborating with peers towards a common goal; adapting language and communicative style to engage with younger mates; devising and designing a tangible object, and planning its creation; practically producing a tangible object identifying, getting and using the proper materials and techniques. In this paper we are mainly interested in reporting and discussing the findings concerning the CT skills, and we will consider the soft skills mentioned above as side learning goals only. For the trainees no learning goals were actually set, since they were involved in the activities only for two hours. However the training was useful to them as it gave them the opportunity to face the Bebras tasklets in a even more friendly setting than the online platform. A very recent work [10 describes a similar project conducted in Lithuania where game cards inspired by Bebras tasklets were proposed outside the challenge to foster CT. In this project too, tangible objects, mostly provided by the teacher, were used to support pupils in their solving process.

\subsection{Methodology}

During the whole project the Teacher acted more as a facilitator of the learning process than as an instructor, keeping in mind the constructivist view of learning: learners actively construct their knowledge and skills through reorganization of their previously acquired mental structure [15]. According to socialconstructivism such construction of knowledge is also guided and influenced by the social context, and thus by the interactions with others and in particular by their use of language [16. Hence the learning process is fostered by feedback, 
examples, and scaffolding by teachers, and interaction with peers rather than only by free exploration [14.

For this project, the Teacher designed the activities in a CSSC (Constructive, Self-Regulated, Situated, and Collaborative) learning environment 11: the first phase required a mindful and effortful involvement by pupils in the exploration of the tasklets and allowed them to individually construct knowledge and meaning (constructive); pupils worked in pairs, exchanging ideas and mediating different points of view (collaborative); during the training sessions pupils acted in a social and cultural context, where learning was further enacted in the interaction with the younger pupils (situated); during the whole project the pupils were let free to decide how to use their time and how to plan their activities, while the Teacher monitored their work giving some feedback, avoiding to give direct instructions if not asked by the pupils themselves, and was available to support them upon request (self-regulated). The Teacher set some constraints in order to promote the expected learning outcomes in CT.

- Pupils were requested to design a game inspired by one among the tasklets they had not correctly solved in the first place. Hence, in order to design their game, they needed to examine the chosen task with care, understanding what it asked the solver to do, and how the correct answer could be found.

- In the transposition toward a tangible game, they were allowed to change some details and introduce variants to the original task, but the resulting game had to reflect the spirit of the original task and in particular be suitable to stimulate the same abilities.

- The rules and directions for the games had to be written in full on a poster to be made available to trainees.

- Pairs were broken up during the two training sessions so that each trainer was in charge of personally conducting the game in one of them.

At the beginning of the first phase, pupils were randomly split in pairs. As one could expect, several pairs were therefore composed by pupils with different levels of cognitive, linguistic, creative, and practical skills, and some of them were actually unhappy of the draw's outcome. This choice was discussed with the pupils and then motivated by the Teacher: they would be asked to collaborate with their mates as true professionals; moreover, identifying at least one positive trait in their mate was a target they had to achieve throughout the project.

During the training sessions (two hour long each), several table islands were arranged in the classroom where the games were displayed, and the trainees could move around the islands to play the games; each trainee was equipped with a card where trainers logged the participation to their games. During the games, the trainees could read the poster with the rules and directions for a game, however trainers were not forbidden to read aloud or explain in their own words the rules for the trainees, or more generally to interact with them.

To assess the project we took into consideration both the products of the pupils involved - namely the tangible games they built - and the overall learning process, focusing mainly on their interactions with peers, trainees, and their 
Teacher. In particular, the Expert attended the second (and last) training session as a non-participant observer. The observation goal was to detect whether and how the CT aspects underlying the Bebras tasklets had been grasped by the trainers. Both the Teacher during the first phase of the project, and the Expert during the last training session used the anecdotal records methodology 12, which consists of short descriptions of behavior as observed in specific situations. As typical in anecdotal records, the observed incidents have then been interpreted, and recommendations arising from the observations have been suggested and, in some cases, immediately implemented.

\section{Process and products}

The 22 pupils in the 6 th grade class were randomly split into 11 pairs. Three pairs chose tasklet "Birthday party" (2018-RO-06), two pairs chose tasklet "Drawing Game" (2018-PK-01), the other pairs chose one of the following tasklets: "Balls" (2017-RS-02), "Board jumps" (2018-CA-06), "Waiter" (2018-IT-06), "Room sharing" (2018-DE-07), "Finding the route" (2018-CY-04), "Dustmen robots" (2018-SK-05) 4 . Ten pairs succeeded in building their games. Most games were realized as posters (some of the posters are shown in Figures 11- 5), and some of them also had mobile elements so that the interactivity of the original tasks could be simulated. One pair only (who chose "Birthday party") did not succeed in getting the game ready for the training sessions. Two pairs added some difficulties in their games that were not included in the original tasks (namely "Drawing Game" and "Birthday party"). Since such difficulties were deemed by the teacher too hard for 3rd graders, and other groups had chosen the same tasks, at the end only the latter games were used in the training sessions. During the training sessions most pairs were split, so that half of the 6th graders attended the first session and the other half attended the second session, and most trainers ran their game individually. Some exceptions occurred: some pairs were kept as such, mainly due to the presence of pupils with special needs; moreover, since "Drawing Game" was chosen by two pairs, but only one version of the game was suitable for the training session, the trainers did not work alone but were coupled with a member of the other pair.

In the following we give some details on the tasklets chosen and how they were used by the pupils. Due to space constraints we limit the description to five tasklets only, namely those which led to situations offering more elements for the discussion. We relate the tasklets to the CT skills we listed in Section 2.1 and we describe how the related games were realized and which differences pupils introduced with respect to the original tasklets. We also report and discuss the relevant incidents that occurred during the first phase and/or the training

\footnotetext{
${ }^{4}$ We report in bracket the international Bebras id code, although the tasklets were sometimes modified to exploit the interactivity potential of the Italian Bebras Platform and to take into account that the Italian contest is team based (whereas it is individual in most of the countries). The screenshots of the actual tasklets (translated to English for this paper) are given in Appendix A
} 
sessions. These facts shed lights on both the cases where pupils had understood the CT concepts, and the cases where critical issues came up.

\subsection{Birthday party}

Tasklet. The tasklet, shown in Figure 1, asks the solver to consider the friendship relationship among animals and place the animales around tables respecting some given constraints, thus it relates to the "Reason" skill. While reading the text one can start placing the animals, considering one constraint at a time, but there is some freedom in the choice of the tables, since some placements are fully determined only by forthcoming constraints. In particular, two critical situations might happen: i. if one places the Rabbit (the first mentioned animal) at the wrong table, she gets eventually stuck due to the different number of seats at the two tables; ii. all animals are placed before the text is over complying with all but the last constraint, but the placement might turn out not to satisfy the last constraint. In both cases, the solver needs to backtrack and reconsider the solution or start over.

Game. The trainers drew the two tables on the poster, and pieces of Velcro tape were stuck around each table as placeholders for the guests. The faces of the animals were drawn on cards, with Velcro on the back, so that they could be attached to the places around the tables. The cards were stored in an envelope attached to the poster. The task's text and the question were written on separate sheets and put in another envelope.

Anecdotal record. At the beginning of the training session, trainers were very directive and basically guided the trainees step by step; when trainers read a sentence like "animal $A$ is friends with animal $B$ ", they also added a comment like "then you have to put animal $A$ at the same table as animal $B$ ", often pointing at the corresponding card. Moreover, when trainees got stuck because of the wrong choice of the table for the Rabbit (case i. above), they straightforwardly suggested to start over by placing it at the other table. Also, as soon as the trainees placed the last animal at a table, the trainers stopped the trainees (even though the text was not over yet) asked them to check the obtained situation against the solution in the envelope, and made them start over when they differed. In other terms, when something went wrong, the trainers did not wait for the trainees to discover the problem and try to fix it by themselves. The Teacher advised the trainers to be more patient, but they appeared skeptical and indeed showed again the tendency of being too directive. After a second intervention, they succeeded in being more patient and realized that the trainees were actually able to eventually detect the inconsistencies by themselves without checking the solution, if allowed to conclude reading the text. Once, a trainee got stuck but refused to start over, asking instead try to fix the solution alone. He switched the tables and then re-checked that all constraints were satisfied. The trainers were really surprised that the trainee was actually able to fix the error by a different approach: "he did find the correct solution, but he found it without starting over!". 
Discussion. Trainers were able to reason on the task's constraints, identifying the correct solution. They also showed to be aware of the two critical situations that might happen. However, the fact that they stopped trainees prematurely suggests that they probably did not identify the origin of the issue for case ii). The last trainee described above showed good abstraction skills, being able to grasp that the relation "being at the same table with" is more important -in this tasklet- than the property "being seated at a certain table". Moreover, this interaction helped the trainers understand an important CT aspect: the same output or final result can be obtained with different strategies.

\subsection{Drawing Game}

Tasklet. The tasklet, shown in Figure 2, is basically a programming question -the programs' goal being to draw shapes- and it relates to "Implement" and "Algo_think" skills. Due to the presence of multiple choices, the tasklet could be solved simply by executing the programs and checking if the obtained output is a square.

Game. The tasklet text was directly written on the poster, but the examples shown in the original were omitted. Moreover, the original tasklet presented a multiple choice question; the trainers opted instead for a constructive question, asking the solver to build a program with the provided commands. In an envelope attached to the poster, some white sheets were made available to write down the program, with lines numbered 1 to 6 , to suggest that six commands were needed in the program.

Anecdotal record. During the preparation of the game, the Teacher suggested that trainees would have benefited from manipulating some tangible objects when creating the program. Trainers were skeptical at first but then they used some cardboard to make four logs and a flag, so that the target drawing could actually be composed with objects. During the first training session, the trainers realized that writing the program on the sheets of paper was too time consuming. Hence, before the next training session, the trainers added some cards with the four available commands, that could be put in a sequence to form a program. During the next training session, however, they observed that multiple copies of each command were actually needed, since some commands had to be used more than once in the same program.

Discussion. The game resulted in a much more difficult task than the original one; according to the Bloom's taxonomy, the required cognitive skill moved from "apply" to "create". This fact suggests that the trainers mastered the CT content implied by the tasklet. During the training, they further realized the importance of re-using the same command more than once and in different positions. This is a peculiar aspect of programming, that does not often appear in other mathematical or logical tasks that require rearranging (e.g., sequencing) 
objects. Moreover, while supporting their trainees struggling in the programwriting process, the trainers came to understand better what is implied in such process: on the one hand one has to select and properly position the appropriate commands (write code), on the other hand one has to simultaneously simulate and trace the effect of the commands themselves (execute code).

\subsection{Balls}

Tasklet. The tasklet, presented in Figure 3 focuses on LIFO stacks, and it relates to "Algo_think" and "Organize" skills.

Game. The ramps with holes and pins were drawn directly on the poster together with the tasklet's text and some new explanations on how the system was supposed to work. As in the original task, four possible alternative answers were given. An extra sheet with the ramp and the holes drawn on it was made available to track the rolling balls. The original number of balls was doubled from 10 to 20 , the number of holes was increased from three to four with their capacity always of decreasing size.

Anecdotal record. During the first phase, the Teacher suggested to prepare some tangible elements to simulate the process. The trainers did not accept the suggestion since "it will make the game too easy". Then the Teacher suggested that they could provide the trainees with some support to trace the process; they agreed but were not able to find a way. At the end, the Teacher suggested to draw the ramps with holes and pins on separate sheets, to be freely sketched by the trainees. During the first training session, the trainers did not use the sheets until after some iterations, when they saw trainees struggling with the task. At the end of the first training session, the trainer commented that the sheets were in fact useful. The trainer of the second session was very patient and intervened only when the trainees were stuck or made some errors in the executions of steps, by re-reading them the appropriate specification when needed, and advising them to trace the process step by step on the sheet.

Discussion. The fact that the trainer of the second session was so precise in reminding the relevant specifications at the exact moment they were needed is evidence that she had understood and remembered how the system worked and was perfectly able to follow the evolution of the system and apply the general specifications to its current state. The latter one is an important CT skill, especially useful when analyzing or debugging programs or systems. Moreover, her manner of supporting the trainees helped them in appreciating how the system evolved in a deterministic way, according to the specifications.

\subsection{Waiter}

Tasklet. The tasklet, presented in Figure 4, asks pupils to choose among different representations (notes) of an order taken by a waiter. The tasklet aimed at 
promoting a reflection on how different ways of representing the same data can serve different purposes, hence it relates to "Organize" and "Represent" skills.

Game. The tasklet text was written on the poster, whereas the multiple choice answers were written on separate sheets and glued to the poster. Each answer had a piece of Velcro tape on top, and a Velcro cross should be used to select the right answer. The setting was changed with respect to the original tasklet: instead of a waiter, the main character was a shop assistant selling make-up products. The game presented only three multiple choice options instead of five. Moreover, the three options in the new setting did not present the same features as the original ones. In fact, each original option contained different pieces of information, whereas the new ones were actually equivalent with respect to the information they contained, differing only in the notation (numbers instead of letters, abbreviations, ...).

Anecdotal record. During the training session, the game was easily solved by all trainees who simply had to pick the shortest option. When the Teacher asked the trainer to explain why they had changed the tasklet, the trainer only focused on the setting and not on the options and the differences in their information content (in fact, there were no differences). Only after recovering the original tasklet on the Bebras Platform and asking to check the correspondence between the options, the trainer realized that he was not able to match the modified ones with the original ones.

Discussion. Most likely, the trainers were able to identify the correct answer by simply recognizing the typical form of waiter's note, but they appeared to have missed the CT content in the explanation and comments to the tasklet. This interpretation is supported by the fact that the correct answer was faithfully transposed in the new setting, whereas the other options did not show the same features as the original ones. For instance, the explanation highlighted that the second option clustered the ordered items into two groups (Drinks and Foods) and could be useful if the service was prepared by two different people in charge respectively of drinks and foods only. This difference totally disappeared in the new setting.

\subsection{Board Jumps}

Tasklet. The tasklet, presented in Figure 5, models a setting with pointers or jump instructions, and it relates to "Algo_think" skill.

Game. The game was built by using shoe boxes. Each of them was labeled, in order, with a capital letter from $A$ to $H$, and a card with the instruction was glued inside each box. No poster was prepared and the tasklet text was written on a piece of paper. The correct solution was written on another sheet and put into an envelope. The text was changed; the basic instruction "2L means to open the box that is 2 positions to the Left" was rewritten as "move this box 
two positions to the left". Notice that this rule is not precise enough, since it is not clear what "move to another position" means, given that a box can be placed next to other boxes but cannot replace another box. Also, this new kind of rules does not fully determine the process, since it is not specified which box must be opened next. It was actually assumed that the boxes be opened in alphabetical order.

The question was changed too, in that solvers were asked to establish how the boxes are sorted at the end of the process. Notice that the new interpretation of the text changes completely the process and its outcome, since the solution of the original tasklet cannot be obtained following the text of the new game. However, the solution in the envelope reported exactly the correct solution of the original tasklet.

Anecdotal record. During the training session, the trainer read aloud the tasklet's text, then waited for the trainees to start, by opening and moving the box labeled $A$. At this point the trainees were stuck and they asked what to do next, thus the trainer explained to follow the alphabetical order, i.e., to open next the box labeled $B$. When the trainees concluded the game, their solutions inevitably differed from the expected one. The trainer then offhandedly explained the fact with vague remarks like "you did not move the boxes into the proper positions". The trainer never tried to repeat the process, showing or checking the proper actions to be carried out to reach the expected outcome. The trainees did not appear convinced but did not engage in any further discussion nor asked explanations.

Discussion. Manifestly, the trainer had not understood the tasklet and in particular the rules, their effect when applied, the overall process, nor the question. Surprisingly, even though the wrong interpretation of the rules and question was not compatible with the correct solution and its explanation (which reported step by step the complete process), the trainer remained comfortably in her interpretation. The trainer's imposing self-assurance on one hand, and the instructions' lack of rigor on the other hand, might have inhibited the critical thinking skills of the trainee. All in all, in this case neither the game preparation nor the training served as promoters of CT skills.

\section{Conclusions and further work}

The idea of asking 6th graders to train younger pupils with Bebras-inspired games they invented has proven to be a good opportunity for situated learning. All but one pair succeeded in finalizing the game they designed: the assignment and the time allocated seem to be well chosen for the age group. In most cases, we collected evidence that the pupils had understood the original tasklets, transposed correctly their core CT ideas, and were able to explain them to their younger mates. The interaction between trainers and trainees shows in more than one case (e.g., "Balls" and "Drawing Game") that trainers were in fact able to 
follow the solving process of the trainees and support them appropriately. Not by chance, these are exactly the cases where the trainers intentionally made the tasklet's task more challenging, which is further evidence of their mastering of the implied CT skills. It is important for the teacher to monitor this aspect and make sure that the implied CT skills are preserved and the level of difficulty is kept adequate for the trainees' age.

With "Birthday party" and "Dustman robots" we also have evidence that the feedback from younger mates helped trainers in further improving their understanding of the task and the important elements therein. Finally, in many cases (e.g., "Room sharing", "Drawing Game", "Dustmen robots"), pupils elaborated the original tasklet content, still preserving its sense and efficacy, showing in the process to be able to identify and abstract the important elements and properties of the entities involved in the tasklet, and the skills required to solve it. However, in two cases (namely "Board jumps" and "The waiter") the trainers clearly did not succeed in understanding the tasklet or the $\mathrm{CT}$ aspects, all the more so to convey them to the younger mates. For these pupils, most activities carried out during the project (and especially the whole training sessions) were not productive, as far as the CT aspects were concerned. At the end of the project, the Teacher reckoned that at first she was not familiar with those tasks and that she focused mainly in building a collaborative atmosphere within the class and in helping pupils with their design and creation of the game. These are probably the reasons why she did not notice at an early stage that the pupils were missing some meaningful points in the tasklets. Considering the results of the project, an important lesson learned by the Teacher is the need to spend some time to deeply examine the chosen tasklets (including the explanation and the commentary) in order to identify more clearly the important elements therein and the underlying $\mathrm{CT}$ content, so as to be able to monitor whether they are grasped during the activities or intervene in case they are not. As for the cross-cutting competencies, the pairs definitely practiced a number of them, e.g., working towards a common goal, designing and building a tangible object, being patient in letting the younger work through the solution of the tasklet without stopping them beforehand, accepting and appreciating other ways of reasoning. We have to point out that initially the trainers displayed some resistance to building and providing trainees with tangible objects to work with, which instead proved to be helpful and appreciated by the younger pupils, as testified also in [10].

All in all, the experience proved that using Bebras tasklets as the social and cultural context for situated learning of CT competencies is indeed quite productive: pupils were generally captivated and showed signals of learning in all the phases. This approach provides many elements to the instructor to monitor the learning process and its effectiveness. Future work aims at measuring and evaluating more analytically the impact of such didactic interventions on improving computational thinking skills. Bebras can in fact be a valuable resource for learning activities, and the richness of the tasklets, sometimes overlooked during the short time available during the contest, is instead fully explored in the deep engaging series of activities such as the ones described here. 


\section{References}

1. International Society for Technology in Education \& Computer Science Teachers Association: Operational definition of computational thinking for K-12 education. http://www.iste.org/docs/ct-documents/ computational-thinking-operational-definition-flyer.pdf (2011)

2. Annalisa Calcagni and Violetta Lonati and Dario Malchiodi and Mattia Monga and Anna Morpurgo: Promoting Computational Thinking Skills: Would You Use this Bebras Task? In: ISSEP 2017. LNCS, vol. 10696, pp. 102--113. Springer (2017). https://doi.org/10.1007/978-3-319-71483-7_9

3. Carlo Bellettini and Fabrizio Carimati and Violetta Lonati and Riccardo Macoratti and Dario Malchiodi and Mattia Monga and Anna Morpurgo: A platform for the Italian Bebras. In: CSEDU 2018 --- Volume 1. pp. 350--357. SCITEPRESS (2018). https://doi.org/10.5220/0006775103500357

4. Carlo Bellettini and Violetta Lonati and Mattia Monga and Anna Morpurgo: How pupils solve online problems: an analytical view. In: CSEDU 2019 --- volume 2. pp. 132--139. SCITEPRESS (2019). https://doi.org/10.5220/0007765801320139

5. Lonati, Violetta and Malchiodi, Dario and Monga, Mattia and Morpurgo, Anna: Is coding the way to go? In: Andrej Brodnik and Jan Vahrenhold (ed.) ISSEP 2015. pp. 165--174 (2015). https://doi.org/10.1007/978-3-319-25396-1_15

6. Corradini, I., Lodi, M., Nardelli, E.: Conceptions and misconceptions about computational thinking among Italian primary school teachers. In: Proc. of the ICER 2017. pp. 136-144. ACM (2017)

7. Dagiene, V.: Supporting computer science education through competitions. In: Proc. 9th WCCE 2009, Education and Technology for a Better World (2009)

8. Dagienè, V., Sentance, S.: It's computational thinking! Bebras tasks in the curriculum. In: Proc. of ISSEP 2016. pp. 28-39. Springer (2016)

9. Dagienè, V., Stupuriene, G.: Informatics education based on solving attractive tasks through a contest. In: KEYCIT 2014. pp. 97-115 (2015)

10. Dagienè, V., Futschek, G., Stupurienè, G.: Creativity in solving short tasks for learning computational thinking. Constructivist Foundations 14(3), 382-396 (2019), https://cepa.info/6060

11. De Corte, E.: Constructive, self-regulated, situated, and collaborative learning: An approach for the acquisition of adaptive competence. Journal of Education 192, 33-47 (2012). https://doi.org/10.1177/0022057412192002-307

12. Froehlich, C.P., Hoyt, K.B.: Guidance testing and other student appraisal procedures for teachers and counselors. Science Research Associates (1959)

13. Haberman, B., Cohen, A., Dagienè, V.: The beaver contest: attracting youngsters to study computing. In: Proc. of ITiCSE 2011. pp. 378-378. ACM (2011)

14. Mayer, R.E.: Should there be a three-strike rule against pure discovery learning? American Psycologist 59, 14-19 (2004)

15. Piaget, J.: The child's constructions of reality. Routledge and Kegan Poul (2013)

16. Vygotsky, L.: Mind in Society: Development of Higher Psychological Processes. Cambridge: Harvard University Press (1978) 


\section{A Tasklets screenshots with games}

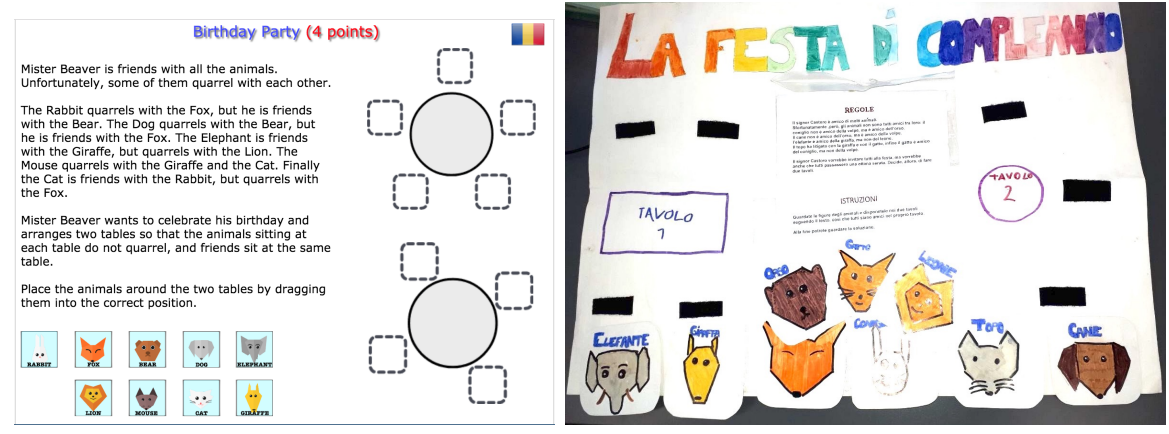

(a) Tasklet

(b) Game

Fig. 1. Birthday Party

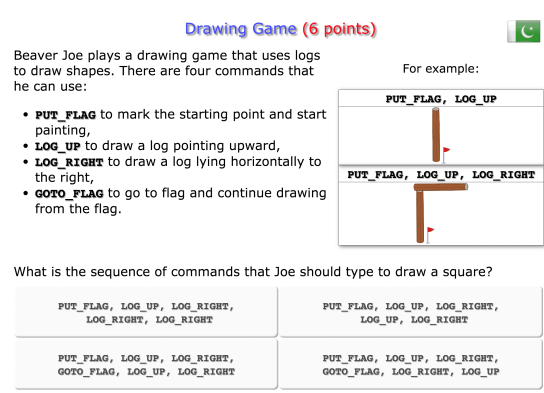

(a) Tasklet

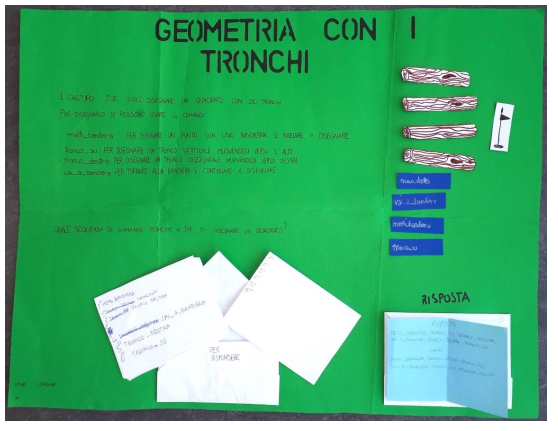

(b) Game

Fig. 2. Drawing Game 


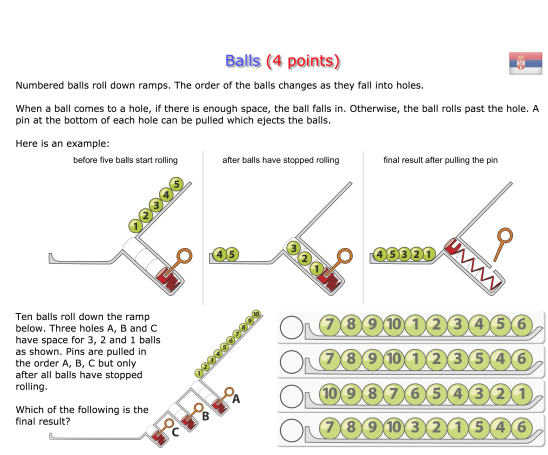

(a) Tasklet

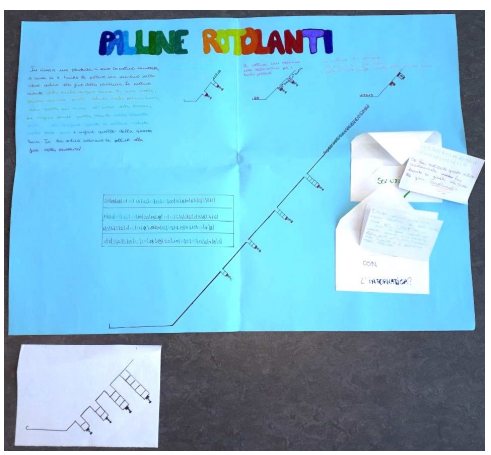

(b) Game

Fig. 3. Balls

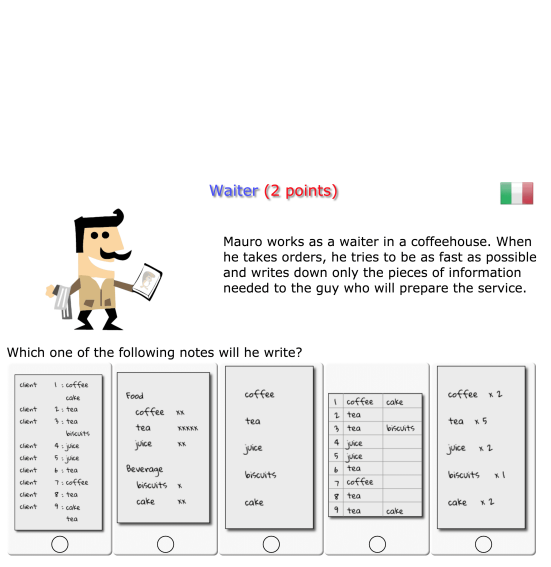

(a) Tasklet

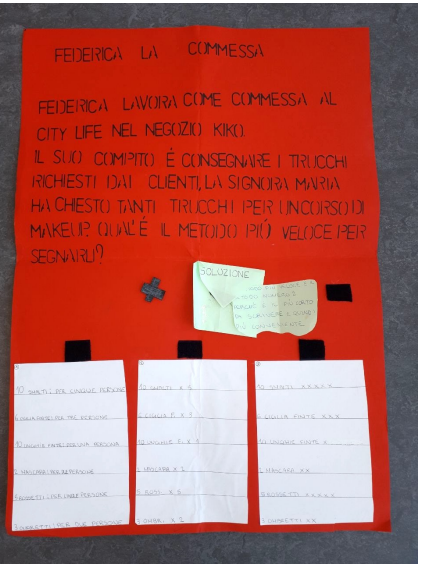

(b) Game

Fig. 4. Waiter

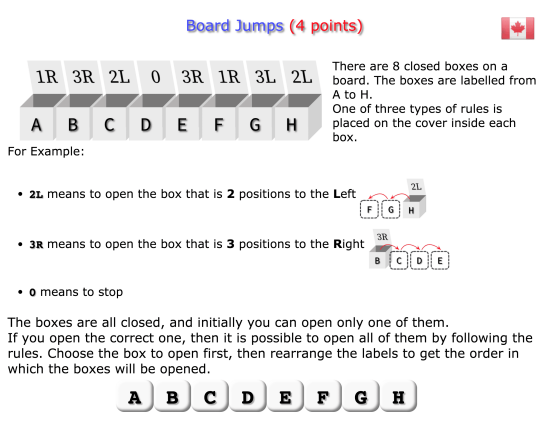

(a) Tasklet

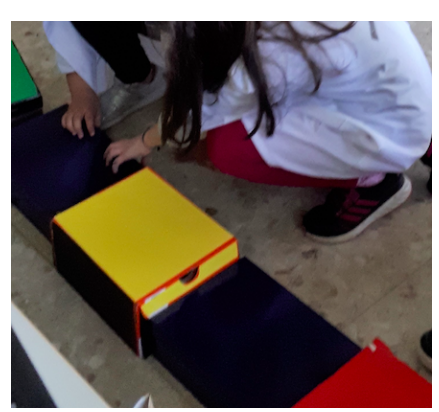

(b) Game

Fig. 5. Board Jumps 